Pacific Journal of Mathematic 


\title{
EQUATIONS WITH OPERATORS FORMING A RIGHT ANGLE
}

\author{
R. E. SHOWALTER
}

The operator $B$ in a complex Hilbert space $H$ is said to form an angle $\theta$ with the (stronger) operator $A$ if $D(A) \subset$ $D(B)$ and, for every $x$ in $D(A),(A x, B x)_{H}$ belongs to the cone $K(\theta)$ of all complex $z$ with $|\arg (z)| \leqq \theta$. If $A$ and $B$ are closed maximal accretive operators and $B$ forms a right angle with $A$, then $A+B$ is closed maximal accretive and the Cauchy problem for each of the equations $u^{\prime}(t)+(A+B) u(t)=f(t)$ and $(I+B) u^{\prime}(t)+A u(t)=f(t)$ is well-posed. Applications to partial differential equations are indicated in the second part.

1. Global perturbations, A linear operator $B: D(B) \rightarrow H, D(B) \subset$ $H$, is accretive if $\operatorname{Re}(B x, x)_{H} \geqq 0$ for all $x \in D(B)$ and maximal accretive if is accretive and has no proper accretive extension in $H$. An accretive operator $B$ is closed if and only if the range $R(I+B)$ is closed. A maximal accretive operator is closed if and only if it has dense domain. For an accretive operator $B, R(I+B)=H$ if and only if $B$ is closed and maximal accretive. These results are given in [6].

Let $B$ be a closed and accretive operator in $H$. Then $R(I+B)$ is closed in $H$ and $I+B$ is a bijection of $D(B)$ onto $R(I+B)$. Hence the set $J \equiv D(B)$ with the inner-product $(x, y)_{J} \equiv((I+B) x,(I+B) y)_{I I}$ is a Hilbert space. $J$ is a subset of $H$ and $(x, x)_{J} \geqq(x, x)_{I I}$ for $x \in J$, so $J$ is topologically imbedded in $H$.

Let $A$ be an operator in $H$ and assume $D(A) \subset D(B)$ and $R(A) \subset$ $R(I+B)$. Define by $T \equiv(I+B)^{-1} A: D(A) \rightarrow J$ an operator in $J$.

\section{Lemma 1. If $A$ is closed then $T$ is closed.}

Proof. Let $x_{n} \in D(A)$ and $\lim T x_{n}=y, \lim x_{n}=x$ in $J$. These imply $\lim A x_{n}=(I+B) y$ and $\lim x_{n}=x$ in $H$, respectively, so the result follows.

Lemma 2. If $T$ is accretive in $J$ and $R(I+A) \supset R(I+B)$, then $R(I+T)$ is dense in $J$.

Proof. Let $x \in J$ be orthogonal to $R(I+T)$ and choose $z \in D(A)$ such that $(I+A) z=(I+B) x$. Then $0=\operatorname{Re}(x,(I+T) z)_{J}=\operatorname{Re}((I+A) z$, $(I+B+A) z)_{H}=|(I+A) z|_{I I}^{2}+\operatorname{Re}(T z, z)_{J}+\operatorname{Re}(z, B z)_{H}-\operatorname{Re}(A z, z)_{I I}$. This implies $\operatorname{Re}(A z, z)_{H} \geqq|(I+A) z|_{H}^{2}$ and thus $0 \geqq|z|^{2}+\operatorname{Re}(A z, z)_{H}+$ 


\section{$|A z|_{H}^{2}$, so $(I+A) z=(I+B) x=0$. Hence $x=0$.}

THEOREM 1. Let $B$ be a closed accretive operator in $H, A$ a closed operator with $R(A) \subset R(1+B) \subset R(1+A)$. Assume $I+B$ forms a right angle with $A$ : $\operatorname{Re}(A x,(I+B) x)_{H} \geqq 0$ for all $x \in D(A) \subset D(B)$. Then $T$ is closed maximal accretive on $J$ and $R(I+B+A)=R(I+B)$.

Proof. The right angle condition is precisely the statement that $T$ is accretive on $J$. Lemma 1 implies $R(I+T)$ is closed and hence (by Lemma 2) equal to $J$.

CoRollary 1. Let $B$ be a closed maximal accretive operator in $H, A$ a closed operator with $R(I+A)=H$, and assume $I+B$ forms a right angle with $A$. Then $R(I+B+A)=H$. If $A$ is accretive (hence, maximal accretive) then $B+A$ is closed maximal accretive.

We note here that if any two of the following three conditions hold, then so does the third: $B$ forms a right angle with $A, A$ is accretive ( $I$ forms a right angle with $A$ ), $I+\alpha B$ forms a right angle with $A$ for every $\alpha>0$. In particular the Corollary 1 is close to a result of [4].

The closed maximal accretive operators are characterized as the negatives of infinitesimal generators of strongly-continuous semigroups of contractions, so Corollary 1 gives a sufficient condition for the wellposedness of a Cauchy problem [5].

CoRollary 2. Let $A$ and $B$ be closed maximal accretive operators on $H$ and assume $I+B$ forms a right angle with $A$. For each $u_{0} \in$ $D(A)$ and continuously differentiable $f:[0, \infty) \rightarrow H$, there is a unique continuously differentiable $u:[0, \infty) \rightarrow H$ with $u(0)=u_{0}, u(t) \in D(A)$ for $t>0$ and

$$
u^{\prime}(t)+(A+B) u(t)=f(t)
$$

This is a perturbation of the Cauchy problem for the equation

$$
u^{\prime}(t)+A u(t)=f(t)
$$

by an (unbounded) operator $B$ which is weaker than $A$ [2]. This result is known to hold when $B$ is replaced by a strongly continuously differentiable map $t \rightarrow B(t)$ of $[0, \infty)$ into the space of continuous linear operators on $H$ [5]. Thus the term $B(t) x(t)$ can be added to (1) and a well-posed problem is obtained. Perturbations of a "local" type are known without our right angle condition $[1,2,4]$. See $[1,3,6]$ for applications of (1) to parabolic and hyperbolic differential equa- 
tions.

In the proof of Theorem 1 we showed that $T$ is closed maximal accretive on $J$, so $-T$ generates a strongly continuous semigroup of contractions on $J$. This yields the following result.

CoROLlary 3. Let $B$ be a closed accretive operator in $H, A$ a closed operator with $R(A) \subset R(I+B) \subset R(I+A)$. Assume $I+B$ forms a right angle with $A$. Then for $u_{0} \in D(A)$, continuously differentiable $f:[0, \infty) \rightarrow H$ and strongly continuously differentiable $B(\cdot)$ from $[0, \infty)$ to the space of continuous linear operators from $J$ to $H$, there is a unique continuously differentiable $u:[0, \infty) \rightarrow J$ with $u(0)=u_{0}$, $u(t) \in D(A)$ and $u^{\prime}(t) \in D(B)$ for $t>0$ and

$$
u^{\prime}(t)+B u^{\prime}(t)+A u(t)+B(t) u(t)=f(t) .
$$

Proof. It suffices to note that (3) is equivalent to the equation $u^{\prime}(t)+T u(t)+(I+B)^{-1} B(t) u(t)=(I+B)^{-1} f(t)$.

The equation (3) arises in applications wherein $B=c A, A$ is a realization of partial differential operator in spatial variables, and $c$ is a complex number [7,9]. Our hypotheses hold if $A$ is a closed accretive operator and $\operatorname{Re}(c) \geqq 0$.

Our second major result is a refinement of Theorem 1 under the (stronger) hypothesis that $I+B$ forms an acute angle with $A$.

THEOREM 2. Let $B$ be a closed accretive operator in $H, A$ a closed operator with $R(A) \subset R(I+B) \subset R(I+A)$. Assume $I+B$ forms an angle $\theta<\pi / 2$ with $A$. Then $-T$ generates an analytic semigroup on $J$.

Proof. Since $T$ is closed maximal accretive, $(\lambda+T)^{-1}$ is in the space $\mathscr{L}(J)$ of bounded linear maps on $J$ and $\left\|(\lambda+T)^{-1}\right\| \leqq(\operatorname{Re}(\lambda))^{-1}$ whenever $\operatorname{Re}(\lambda)>0$. It suffices to show that the operators $\left\{\lambda(\lambda+T)^{-1}\right.$ : $\operatorname{Re}(\lambda)>0\}$ are uniformly bounded in $\mathscr{L}(J)$ [10].

The acute angle assumption implies the existence of a $k>0$ such that

$$
\operatorname{Re}(T x, x)_{J} \geqq k\left|\operatorname{Im}(T x, x)_{J}\right|, x \in D(A),
$$

and we may assume $k \leqq 1$. Letting $\lambda=\sigma+i \tau, \sigma>0$, and $x \in D(A)$ we have

$$
\operatorname{Re}((\lambda+T) x, x)_{J}=\sigma(x, x)_{J}+\operatorname{Re}(T x, x)_{J}
$$

and

$$
\left|\operatorname{Im}((\lambda+T) x, x)_{J}\right| \geqq|\tau|(x, x)_{J}-\left|\operatorname{Im}(T x, x)_{J}\right|
$$


If it were not true that

$$
\left|\operatorname{Im}((\lambda+T) x, x)_{J}\right| \geqq(|\tau| / 2)(x, x)_{J},
$$

then from (4), (6) and the negation of (7) we have

$$
\operatorname{Re}(T x, x)_{J} \geqq(k|\tau| / 2)(x, x)_{J} \cdot
$$

Thus, at least one of (7), (8) holds and this gives

$$
\left|((\lambda+T) x, x)_{J}\right| \geqq(k|\tau| / 2)(x, x)_{J} \cdot
$$

From this last estimate follows the inequality

$$
\left\|(\lambda+T)^{-1}\right\| \leqq(2 / k|\tau|) .
$$

But we already have this quantity bounded by $(1 / \sigma)$ (cf. (5)), so we obtain finally,

$$
\left\|\lambda(\lambda+T)^{-1}\right\| \leqq 4 / k, \operatorname{Re}(\lambda)>0
$$

CoRollary. For each $u_{0} \in D(B)$ and Hölder continuous $f:[0, \infty) \rightarrow$ $H$, there is a unique continuously differentiable $u:[0, \infty) \rightarrow H$ for which $u(0)=u_{0}, u(t) \in D(A)$ for $t>0$ and (3) is satisfied [1, 2].

2. Applications. The applications of the abstract Cauchy problem for (2) are well known [1, 3, 6] so we shall restrict our discussion to applications of (3). No attempt will be made to be comprehensive in any sense, but we shall give three elementary examples for which generalizations are obvious.

Let $H=L^{2}(0,1)$, the Lebesgue square-summable (equivalence classes of) functions on the unit interval, and let $H^{k}(0,1)$ be the Sobolev space of elements of $L^{2}(0,1)$ whose derivatives through order $k$ are in $L^{2}(0,1)$ [1]. Let $c$ be a complex number with $|c| \leqq 1$ and define $B=d / d x$ on $D(B)=\left\{\phi \in H^{1}(0,1): \phi(0)=c \phi(1)\right\}$. Then $B$ is closed maximal accretive in $H$. Let $A \equiv B$; then we have

$$
\operatorname{Re}(A \dot{\phi},(I+B) \dot{\phi})_{H}=\left(1-|c|^{2}\right)\left|\phi^{\prime}(1)\right|^{2} / 2+\int_{0}^{1}\left|\phi^{\prime}\right|^{2}
$$

for $\phi \in D(A)=D(B)$, so $I+B$ forms a right angle with $A . \quad J \equiv D(B)$ is a closed subspace of $H^{1}(0,1)$, so we may define $B(t): J \rightarrow H$ by $B(t) \phi=b_{1}(t) \phi^{\prime}+b^{2}(t) \phi . \quad B(\cdot)$ is strongly continuously differentiable if $b_{1}$ and $b_{2}$ are continuously differentiable. Finally, let $F$ be continuously differentiable on $[0,1] \times[0, \infty)$ and define $f(t)=F(\cdot, t)$. Then $f$ is continuously differentiable from $[0, \infty)$ to $H$. Thus, Corollary 3 implies that for each $u_{0} \in D(B)$ there is a unique (generalized) solution $u(x, t)$ of 


$$
u_{t}+u_{x t}+u_{x}+b_{1}(t) u_{x}+b_{2}(t) u=F
$$

in $(0,1) \times(0, \infty)$ for which $u(x, 0)=u_{0}(x)$ and $u(0, t)=c u(1, t)$ for $t \geqq$ 0 . Thus our results apply to the hyperbolic equation (9) with boundary conditions specified on the characteristics. Furthermore, we can use the Poincaré inequality

$$
\int_{0}^{1}|\phi|^{2} \leqq 2|\phi(1)|^{2}+4 \int_{0}^{1}\left|\phi^{\prime}\right|^{2}, \phi \in H^{1}(0,1)
$$

to show that $I+B$ forms an acute angle with $A$ when $|c|<1$. This permits us to relax the smoothness requirements on $B(t)$ and $f(t)$ in (9).

For our second example we take $H, B, B(t)$ and $f(t)$ as above and define $A \equiv-(d / d x)^{2}$ on $D(A)=\left\{\phi \in H^{2}(0,1): \phi(0)=c \phi(1), \bar{c} \phi^{\prime}(0)=\phi^{\prime}(1)\right\}$. Then $A$ is closed maximal accretive in $H$ and

$$
\operatorname{Re}(A \phi,(I+B) \phi)=\int_{0}^{1}\left|\phi^{\prime}\right|^{2}+\left|\phi^{\prime}(0)\right|^{2}\left(1-|c|^{2}\right) / 2,
$$

for $\phi \in D(A) \subset D(B)$, so $I+B$ forms a right angle with $A$. As before, we have for each $u_{0} \in D(A)$ a unique solution of

$$
u_{t}+u_{x t}+b_{1}(t) u_{x}+b_{2}(t) u=F
$$

in $(0,1) \times(0, \infty)$ for which $u(x, 0)=u_{0}(x)$ and $u(0, t)=c u(1, t), \bar{c} u_{x}(0, t)=$ $u_{x}(1, t)$ for $t \geqq 0$. We cannot improve the result to obtain an acute angle above, but this is expected since we would then have a regularity result (see below) too strong for the hyperbolic equation (10).

For our final example let $G$ be a bounded open subset of $\boldsymbol{R}^{n}$ with $G$ on one side of its infinitely differentiable boundary $\partial G . H^{k}(G)$ is the Sobolev space of (equivalence classes of) functions all of whose derivatives through order $k$ are in $L^{2}(G)$. Let $\Delta \equiv \sum_{i=1}^{n}\left(\partial / \partial x_{i}\right)^{2}$ be the Laplacian operator on the domain $D(\Delta)=\left\{\phi \in H^{2}(G): \phi=0\right.$ on $\left.\partial G\right\}$. Then for each complex $b$ with $\operatorname{Re}(b) \geqq 0$, the operator $B \equiv-b \Delta$ with $D(B)=D(\Delta)$ if $b \neq 0$ and $D(B)=H$ if $b=0$ is closed maximal accretive in $H \equiv L^{2}(G)$. Let $\operatorname{Re}(a) \geqq 0$ for the nonzero complex number $a$ and define $A \equiv a \Delta^{2}$ on $D(A) \equiv\left\{\phi \in H^{4}(G): \phi=\Delta \phi=0\right.$ on $\left.\partial G\right\}$. Then $A$ is closed maximal accretive in $H$. From the divergence theorem we obtain

$$
(A \phi,(I+B) \phi)_{H}=a(\Delta \phi, \Delta \phi)_{H}=a \bar{b} \int_{G} \Sigma\left|\partial \Delta \phi / \partial x_{i}\right|^{2}
$$

for $\phi \in D(A)$. Thus $I+B$ forms a right (acute) angle with $A$ if $\operatorname{Re}(a \bar{b}) \geqq$ 0 (resp., $\operatorname{Re}(a \bar{b})>0$ and $\operatorname{Re}(a)>0$ ), and for each $u_{0} \in D(A)$ (resp., $u_{0} \in D(B)$ ) there is a unique (generalized) solution of 


$$
u_{t}-b \Delta u_{t}+a \Delta^{2} u=0
$$

in $G \times(0, \infty)$ for which $u(x, 0)=u_{0}(x)$ and $u(x, t)=\Delta u(x, t)=0$ for $x \in \partial G$ and $t>0$. Nonhomogeneous terms and perturbations by first order spatial derivatives can be added to (11) when $b \neq 0$. When Theorem 2 applies, the solution of (3) with $B(t)=f(t)=0$ belongs to the domain of every power of the generator $-T$. Hence, when $1-b \Delta$ forms an acute angle with $a \Delta^{2}$, the solution $u(t)=u(x, t)$ of (11) belongs to $\left((1-b \Delta)^{-1} a \Delta^{2}\right)^{-n}[D(A)] \subset H^{2 n+2}(G)$ for every $t>0$ and $n>0$. Thus $u(x, t)$ is by Sobolev's lemma a $C^{\infty}$ function of $x$. Further, one can show by standard techniques $[1,3,8]$ that $u(x, t)$ is infinitely differentiable in $x$ and $t$ and is a genuine (pointwise) solution of (11).

The last example illustrates the technique when $A$ is a polynomial with coefficients in the right half-plane in a self-adjoint operator $B$.

\section{REFERENCES}

1. R. Carroll, Abstract Methods in Partial Differential Equations, Harper and Row, New York, 1969.

2. T. Kato, Perturbation Theory for Linear Operators, Springer-Verlag, Berlin, 1966,

3. P. Lax and A. Malgram, "Parabolic equations," Contributions to the Theory of Partial Differential Equations, Ann. Math. Studies, No. 33, Princeton, N. J., Princeton University Press, (1954), 167-190.

4. N. Okazawa, Two perturbation theorems for contraction semigroups in a Hilbert space, Proc. Japan Acad., 45 (1969), 850-853.

5. R. S. Phillips, Perturbation theory for semi-groups of linear operators, Trans. Amer. Math. Soc., 74 (1954), 199-221.

6. - Dissipative operators and hyperbolic systems of partial differential equations, Trans. Amer. Math. Soc., 90 (1959), 193-254.

7. R. E. Showalter, Partial differential equations of Sobolev-Galpern type, Pacific J. Math., 31 (1969), 787-793.

8. - Local regularity of partial differental equations of Sobolev-Galpern type, Pacific J. Math., (1970), 781-787.

9. W- Well-posed problems for a partial differential equation of order $2 m+1$, SIAM J. Math. Anal., 1 (1970), 214-231.

10. K. Yoshida, Functional Analysis, Springer-Verlag, Berlin, 1965.

Received December 10, 1971.

UNIVERSITY OF TEXAS 


\section{PACIFIC JOURNAL OF MATHEMATICS}

EDITORS

\author{
H. SAMELSON \\ Stanford University \\ Stanford, California 94305 \\ C. R. HOBBY \\ University of Washington \\ Seattle, Washington 98105
}

\author{
J. DUGUNDJI \\ Department of Mathematics \\ University of Southern California \\ Los Angeles, California 90007 \\ RICHARD ARENS \\ University of California \\ Los Angeles, California 90024
}

\section{ASSOCIATE EDITORS}

E. F. BECKENBACH

B. H. NeUManN

F. WOLF

K. YosHIDA

\section{SUPPORTING INSTITUTIONS}

UNIVERSITY OF BRITISH COLUMBIA

CALIFORNIA INSTITUTE OF TECHNOLOGY

UNIVERSITY OF CALIFORNIA

MONTANA STATE UNIVERSITY

UNIVERSITY OF NEVADA

NEW MEXICO STATE UNIVERSITY

OREGON STATE UNIVERSITY

UNIVERSITY OF OREGON

OSAKA UNIVERSITY
UNIVERSITY OF SOUTHERN CALIFORNIA

STANFORD UNIVERSITY

UNIVERSITY OF TOKYO

UNIVERSITY OF UTAH

WASHINGTON STATE UNIVERSITY UNIVERSITY OF WASHINGTON

AMERICAN MATHEMATICAL SOCIETY NAVAL WEAPONS CENTER 


\section{Pacific Journal of Mathematics}

\section{Vol. 45, No. $1 \quad$ September, 1973}

William George Bade, Complementation problems for the Baire classes .......... 1

Ian Douglas Brown, Representation of finitely generated nilpotent groups ........ 13

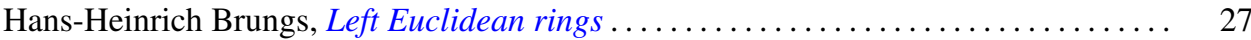

Victor P. Camillo and John Cozzens, A theorem on Noetherian hereditary rings ..... 35

James Cecil Cantrell, Codimension one embeddings of manifolds with locally flat

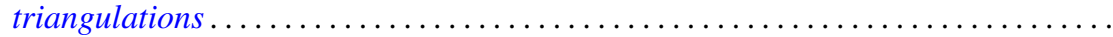

L. Carlitz, Enumeration of up-down permutations by number of rises . . . . . . . . . .

Thomas Ashland Chapman, Surgery and handle straightening in Hilbert cube

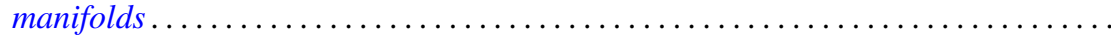

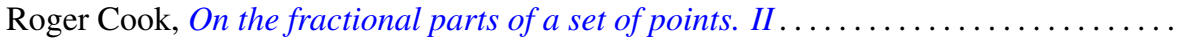

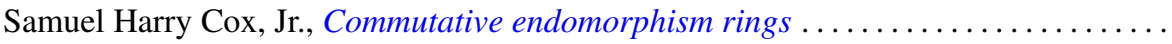

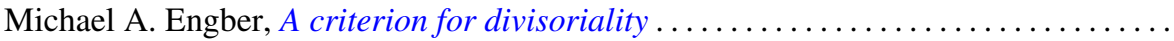

Carl Clifton Faith, When are proper cyclics injective . . . . . . . . . . . . . . 97

David Finkel, Local control and factorization of the focal subgroup . . . . . . . . . 113

Theodore William Gamelin and John Brady Garnett, Bounded approximation by

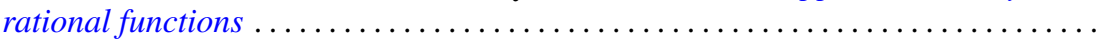

Kazimierz Goebel, On the minimal displacement of points under Lipschitzian

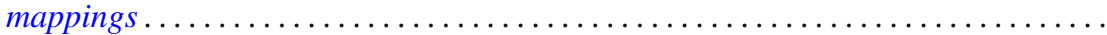

Frederick Paul Greenleaf and Martin Allen Moskowitz, Cyclic vectors for representations associated with positive definite measures: nonseparable

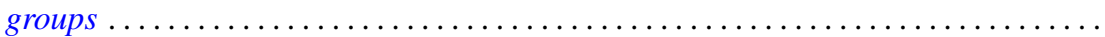

Thomas Guy Hallam and Nelson Onuchic, Asymptotic relations between perturbed linear systems of ordinary differential equations .

David Kent Harrison and Hoyt D. Warner, Infinite primes of fields and completions. .

James Michael Hornell, Divisorial complete intersections . ......

Jan W. Jaworowski, Equivariant extensions of maps ..............

John Jobe, Dendrites, dimension, and the inverse arc function .. .

Gerald William Johnson and David Lee Skoug, Feynman integrals of non-factorable

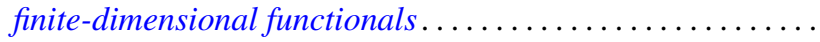

Dong S. Kim, A boundary for the algebras of bounded holomorphic functions ...... 269

Abel Klein, Renormalized products of the generalized free field and its derivatives ... 275

Joseph Michael Lambert, Simultaneous approximation and interpolation in $L_{1}$ and

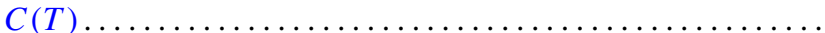

Kelly Denis McKennon, Multipliers of type $(p, p)$ and multipliers of the group

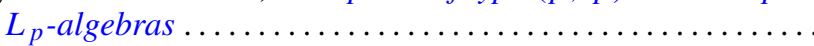

William Charles Nemitz and Thomas Paul Whaley, Varieties of implicative

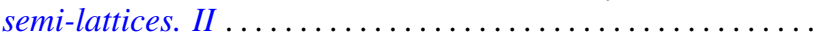

Donald Steven Passman, Some isolated subsets of infinite solvable

Norma Mary Piacun and Li Pi Su, Wallman compactifications on E-completely

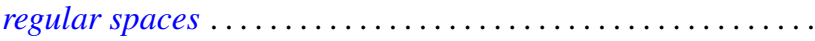

Jack Ray Porter and Charles I. Votaw, $S(\alpha)$ spaces and regular Hausdorff extensions....

Gary Sampson, Two-sided $L_{p}$ estimates of convolution transforms .

Ralph Edwin Showalter, Equations with operators forming a rig
Raymond Earl Smithson, Fixed points in partially ordered sets .

Victor Snaith and John James Ucci, Three remarks on symmetric products and

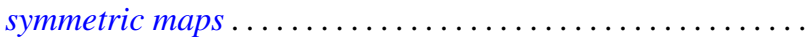

